\title{
RAMSEY MEETS LAIBSON IN THE NEOCLASSICAL GROWTH MODEL*
}

\author{
ROBERT J. BARRO
}

The neoclassical growth model is modified to include a variable rate of time preference. With no commitment ability and log utility, the equilibrium features a constant effective rate of time preference and is observationally equivalent to the standard model. The extended framework yields testable linkages between the extent of commitment ability and the rates of saving and growth. The model also has welfare implications, including the optimal design of institutions that facilitate household commitments. Steady-state results are obtained for general concave utility functions, and some properties of the transitional dynamics are characterized for isoelastic utility.

Many of the basic frameworks in macroeconomics, including the neoclassical growth model, rely on the assumption that households have a constant rate of time preference. However, the rationale for this assumption is unclear. ${ }^{1}$ Perhaps this is because the reason for individuals to have positive time preference is itself unclear.

Ramsey [1928, p. 543] preferred to use a zero rate of time preference. He justified this approach in a normative context by saying "we do not discount later enjoyments in comparison with earlier ones, a practice which is ethically indefensible." Similarly, Fisher [1930, Ch. IV] argued that time preference-or impatience, as he preferred to call it-reflects mainly a person's lack of foresight and self-control. One reason that economists have not embraced a zero rate of time preference is that it causes difficulties for the long-run equilibrium. Thus, most analyses have assumed that the rate of time preference is positive but constant.

As has been known since Strotz [1956] and the elaborations of Pollak [1968] and Goldman [1980]—and understood much earlier

* Basically, David Laibson is responsible for my working on this topic. I thank Christopher Carroll for an insightful discussion and for suggesting something close to the title. The point of the title is that Ramsey was aware of the Strotz-type problem caused by a nonconstant rate of time preference. I have also benefited from comments by Daron Acemoglu, Alberto Alesina, John Campbell, Diego Comin, Roberta Gatti, Edward Glaeser, Elhanan Helpman, Kenneth Judd, N. Gregory Mankiw, Torsten Persson, Jordan Rappaport, Thomas Sargent, Lars Svensson, and Jorgen Weibull. Marios Angeletos provided excellent research assistance.

1. See Koopmans [1960] and Fishburn and Rubinstein [1982] for axiomatic derivations of a constant rate of time preference.

(C) 1999 by the President and Fellows of Harvard College and the Massachusetts Institute of Technology.

The Quarterly Journal of Economics, November 1999 
by Ramsey [1928] ${ }^{2}$-nonconstancy of the rate of time preference can create a time-consistency problem. This problem arises because the relative valuation of utility flows at different dates changes as the planning date evolves. In this context, committed choices of consumption typically differ from those chosen sequentially, taking account of the way that future consumption will be determined. Therefore, the commitment technology matters for the outcomes.

Laibson [1997a, 1997b], motivated partly by introspection and partly by experimental findings, has made compelling observations about ways in which rates of time preference vary. ${ }^{3} \mathrm{He}$ argues that individuals are highly impatient about consuming between today and tomorrow but are much more patient about choices advanced further in the future, for example, between 365 and 366 days from now. Hence, rates of time preference would be very high in the short run but much lower in the long run, as viewed from today's perspective.

Given these insights and evidence, it is important to know whether economists can continue to rely on the neoclassical growth model as their workhorse framework for dynamic macroeconomics. This paper largely answers this question in the affirmative but also brings out various ways in which Laibsonstyle preferences extend the empirical content of the Ramsey model.

\section{Structure of the Neoclassical Growth Model with a VARIABle Rate of Time PREFEREnCE}

In the conventional model, consumer preferences take the form, ${ }^{4}$

$$
U(\tau)=\int_{\tau}^{\infty} u[c(t)] \cdot e^{-\rho \cdot(t-\tau)} d t
$$

2. In the part of his analysis that allows for time preference, Ramsey [1928, p. 439] says "In assuming the rate of discount constant, I [mean that] the present value of an enjoyment at any future date is to be obtained by discounting it at the rate $\rho$.... This is the only assumption we can make, without contradicting our fundamental hypothesis that successive generations are activated by the same system of preferences. For, if we had a varying rate of discount-say a higher one for the first fifty years-our preference for enjoyments in 2000 A.D. over those in 2050 A.D. would be calculated at the lower rate, but that of the people alive in 2000 A.D. would be at the higher."

3. For discussions of the experimental evidence, see Thaler [1981], Ainslie [1992], and Loewenstein and Prelec [1992].

4. For the development of this model, see Ramsey [1928], Cass [1965], Koopmans [1965], and the exposition in Barro and Sala-i-Martin [1995, Ch. 2]. 
where $\tau$ is the current date, $u^{\prime}(c)>0, u^{\prime \prime}(c)<0$, and $\rho>0$ is the constant rate of time preference. Following standard practice, the time discounting for period $t$ depends only on the distance in time, $t-\tau$, from the current date. ${ }^{5}$

The extension here is to modify equation (1) to

$$
U(\tau)=\int_{\tau}^{\infty} u[c(t)] \cdot e^{-[\rho \cdot(t-\tau)+\phi(t-\tau)]} d t,
$$

where $\phi(t-\tau) \geq 0$ brings in the aspects of time preference that cannot be described by the standard exponential factor, $e^{-\rho \cdot(t-\tau)}{ }^{6}$ The new term is assumed, as in the case of the conventional time-preference factor, to depend only on the distance in time, $t-$ $\tau$. We can normalize to have $\phi(0)=0$.

The function $\phi(\cdot)$ is taken in the main discussion to be continuous and twice differentiable. The expression $\rho+\phi^{\prime}(v)$ gives the instantaneous rate of time preference at the time distance $v=t-\tau \geq 0$. The assumed properties, which follow Laibson [1997a], are $\phi^{\prime}(v) \geq 0, \phi^{\prime \prime}(v) \leq 0$, and $\phi^{\prime}(v)$ approaches zero as $v$ tends to infinity. These properties imply that the rate of time preference is high in the near term but roughly constant at the lower value $\rho$ in the distant future.

Consumers with these preferences are impatient about consuming right now, but they need not be shortsighted in the sense of failing to take account of long-term consequences. The present analysis assumes no decision-making failures of this sort. ${ }^{7}$ Moreover, consumer sovereignty suggests that the preferences that feature high short-term impatience ought to be respected for welfare analyses, and the subsequent analysis takes this approach.

The rest of the model is standard. The production function is the usual neoclassical one, given by

$$
y=f(k),
$$

5. The utility expression can be extended without affecting the basic results to include the chronological date $t$ and a household's age and other life-cycle characteristics.

6 . In this formulation, a person at time $\tau$ has preferences that relate directly to the path of $c(t)$. An alternative approach, as in Epstein and Hynes's [1983] recursive preferences, would have $U(\tau)$ depending only on $c(\tau)$ and on utility as evaluated by the "next self" at the next instant in time, $U(\tau+\varepsilon)$. No time inconsistency would arise under this specification. Becker and Barro [1988] use a form of recursive preferences in an intergenerational context.

7. In contrast, Akerlof [1991] assumes that "individuals choose a series of current actions without fully appreciating how those actions will affect future perceptions and behavior." 
where $y$ is output per worker and $k$ is capital per worker, with $f^{\prime}(k)>0$ and $f^{\prime \prime}(k)<0$. The assumption for now is that population is constant and technological progress is nil. The number of workers (and the quantity of labor input) equals the constant population. The economy is closed, so that assets per person equal capital per worker, $k$. The rate of return, $r(t)$, equals $f^{\prime}[(k(t)]-\delta$, where $\delta \geq 0$ is the constant rate of depreciation on capital, and the wage rate, $w(t)$, equals $f[k(t)]-k(t) \cdot f^{\prime}[k(t)]$.

\section{Results UNDER COMMitMent}

The first-order optimization conditions for the representative household's path of consumption, $c(t)$, would be straightforward if the full path of current and future consumption could be chosen in a committed manner at the present time, $\tau$. For example, if utility takes the isoelastic form,

$$
u(c)=\left(c^{1-\theta}-1\right) /(1-\theta),
$$

where $\theta>0$, then the usual Ramsey formula for the growth rate of consumption becomes

$$
\left(\frac{1}{c}\right) \cdot\left(\frac{d c}{d t}\right)=\left(\frac{1}{\theta}\right) \cdot\left[r(t)-\rho-\phi^{\prime}(t-\tau)\right]
$$

for $t>\tau$. The new element is the addition of the term $\phi^{\prime}(t-\tau)$ to $\rho$. Equation (5) can be viewed as coming from usual perturbation arguments, whereby consumption is lowered at some point in time and raised at another point in time-perhaps the next instant in time-with all other values of consumption held constant.

Given the assumed properties for $\phi(\cdot), \rho+\phi^{\prime}(t-\tau)$ would start out at a high value and then decline toward $\rho$ as $t-\tau$ tended toward infinity. Thus, the steady-state rate of time preference would be $\rho$, and the steady-state of the model would coincide with that of the usual model. The new results would involve the transition, during which time-preference rates were greater than $\rho$ but falling over time.

One problem with this solution is that the current time $\tau$ is arbitrary and, in the typical situation, the potential to commit did not suddenly arise at this time. Rather, if perpetual commitments on consumption were feasible, then these commitments would likely have existed in the past, perhaps in the infinite past. In this last situation, current and all future values of consumption would have been determined earlier, and $\tau$ would be effectively minus 
infinity, so that $\phi^{\prime}(t-\tau)$ would be zero for all $t \geq 0$. Hence, the rate of time preference would equal $\rho$ for all $t \geq 0$, and the standard Ramsey results would apply throughout, not just in the steady state.

The more basic problem is that commitment on future choices of $c(t)$ is problematic. The next section therefore works out the solution in the absence of any commitment technology for future consumption. In this setting, the household can determine at time $\tau$ only the instantaneous flow of consumption, $c(\tau)$.

\section{Results Without COMmitment UNDER LOG UTILITY}

The first-order condition in equation (5) will not generally hold without commitment, because it is infeasible for the household to carry out the perturbations that underlie the condition. Specifically, the household cannot commit to lowering $c(\tau)$ at time $\tau$ and increasing $c(t)$ at some future date, while holding fixed consumption at all other dates. Instead, the household has to figure out how its setting of $c(\tau)$ at time $\tau$ will alter its stock of assets and how this change in assets will influence the choices of consumption at later dates.

The full solution without commitment is worked out here for $\log$ utility, $u(c)=\log (c)$. The steady-state results for a general concave utility function are discussed in a later section. Some transitional results under isoelastic utility are derived in a still later section.

Think of choosing $c(t)$ at time $\tau$ as the constant flow $c(\tau)$ over the short discrete interval $[\tau, \tau+\varepsilon]$. The length of the interval, $\varepsilon$, will eventually approach zero and thereby generate results for continuous time. The full integral of utility flows from equation (2) can be broken up into two pieces:

$$
\begin{aligned}
U(\tau)= & \int_{\tau}^{\tau+\varepsilon} \log [c(t)] \cdot e^{-[\rho \cdot(t-\tau)+\phi(t-\tau)]} d t \\
& +\int_{\tau+\varepsilon}^{\infty} \log [c(t)] \cdot e^{-[\rho \cdot(t-\tau)+\phi(t-\tau)]} d t \\
\approx & \varepsilon \cdot \log [c(\tau)]+\boldsymbol{J}_{\tau+\varepsilon}^{\infty} \log [c(t)] \cdot e^{-[\rho \cdot(t-\tau)+\phi(t-\tau)]} d t,
\end{aligned}
$$

where the approximation comes from taking $e^{-[\rho \cdot(t-\tau)+\phi(t-\tau)]}$ as equal to unity over the interval $[\tau, \tau+\varepsilon]$. This approximation will 
become exact in the equilibrium as $\varepsilon$ tends to zero. Note that log utility has been assumed. ${ }^{8}$

The consumer can pick $c(\tau)$ and thereby the choice of saving at time $\tau$. This selection influences $c(t)$ for $t \geq \tau+\varepsilon$ by affecting the stock of assets, $k(\tau+\varepsilon)$, available at time $\tau+\varepsilon$. To determine the optimal $c(\tau)$, the household has to know, first, the relation between $c(\tau)$ and $k(\tau+\varepsilon)$ and, second, the relation between $k(\tau+\varepsilon)$ and the choices of $c(t)$ for $t \geq \tau+\varepsilon$.

The first part of the problem is straightforward. The household's budget constraint is

$$
\frac{d k}{d t}=r(t) \cdot k(t)+w(t)-c(t)
$$

where the economywide prices, $r(t)$ and $w(t)$, are treated as given by the household. For a given starting stock of assets, $k(\tau)$, the stock at time $\tau+\varepsilon$ is determined by

$$
k(\tau+\varepsilon) \approx k(\tau) \cdot[1+\varepsilon \cdot r(\tau)]+\varepsilon \cdot w(\tau)-\varepsilon \cdot c(\tau) .
$$

The approximation comes from neglecting compounding over the interval $(\tau, \tau+\varepsilon)$-that is, from ignoring terms of the order of $\varepsilon^{2}$-and from treating the variables $r(t)$ and $w(t)$ as constants over this interval. These assumptions will be satisfactory in the equilibrium when $\varepsilon$ approaches zero. The important result from equation (8) is that

$$
d[k(\tau+\varepsilon) / d[c(\tau)] \approx-\varepsilon .
$$

Hence, more consumption today means fewer assets at the next moment in time.

The difficult calculation involves the link between $k(\tau+\varepsilon)$ and $c(t)$ for $t \geq \tau+\varepsilon$, that is, the propensities to consume out of assets. In the standard Ramsey model with log utility, $c(t)$ equals the constant fraction $\rho$ of wealth, where wealth equals $k(t)$ plus the present value of wages. The fraction is constant because the income and substitution effects associated with future interest rates exactly cancel under log utility. (See, for example, Barro and Sala-i-Martin [1995, Ch. 2].) Given this background, it is reasonable to conjecture that the income and substitution effects associated with future interest rates would still cancel under log utility, even though the rate of time preference is variable and commit-

8. Pollak [1968, section 2] works out results under log utility with a finite horizon and a zero interest rate. 
ment is absent. However, the constant of proportionality, denoted by $\lambda$, need not equal $\rho$. Thus, the conjecture-which turns out to be correct-is that consumption is given by

$$
c(t)=\lambda \cdot[k(t)+\text { present value of wages }]
$$

for $t \geq \tau+\varepsilon$ for some constant $\lambda>0 .^{9}$

Under the assumed conjecture, it can be verified that $c(t)$ grows at the rate $r(t)-\lambda$ for $t \geq \tau+\varepsilon$. Hence, for any $t \geq \tau+\varepsilon$, consumption is determined from

$$
\log [c(t)]=\log [c(\tau+\varepsilon)]+\int_{\tau+\varepsilon}^{t} r(v) d v-\lambda \cdot(t-\tau-\varepsilon) .
$$

The expression for utility from equation (6) can therefore be written as

$$
U(\tau) \approx \varepsilon \cdot \log [c(\tau)]+\log [c(\tau+\varepsilon)] \cdot \int_{\tau+\varepsilon}^{\infty} e^{-[\rho \cdot(t-\tau)+\phi(t-\tau)]} d t
$$

+ terms that are independent of $c(t)$ path.

Define the integral

$$
\Omega \equiv \int_{0}^{\infty} e^{-[\rho v+\phi(v)]} d v .
$$

This expression, which is constant over time, corresponds, as $\varepsilon$ approaches zero, to the integral in equation (11).

The marginal effect of $c(\tau)$ on $U(\tau)$ can be calculated as

$$
\frac{d[U(\tau)]}{d[c(\tau)]} \approx \frac{\varepsilon}{c(\tau)}+\frac{\Omega}{c(\tau+\varepsilon)} \cdot \frac{d[c(\tau+\varepsilon)]}{d[k(\tau+\varepsilon)]} \cdot \frac{d[k(\tau+\varepsilon)]}{d c(\tau)} .
$$

The final derivative equals $-\varepsilon$, from equation (9), and the next-to-last derivative equals $\lambda$, according to the conjectured solution in equation (10). Therefore, setting $d[U(\tau)] / d[c(\tau)]$ to zero implies that

$$
c(\tau)=[c(\tau+\varepsilon)] / \Omega \lambda .
$$

If the conjectured solution is correct, then $c(\tau+\varepsilon)$ must approach $c(\tau)$ as $\varepsilon$ tends to zero. Otherwise, $c(t)$ would exhibit

9. Phelps and Pollak [1968, section IV] use an analogous conjecture to work out a Cournot-Nash equilibrium for their problem. They assume isoelastic utility and a linear technology, so that the rate of return is constant. The last property is critical, because consumption is not a constant fraction of wealth under isoelastic utility (except in the logarithmic case) if the rate of return varies over time. The linear technology also eliminates any transitional dynamics, so that the economy is always in a position of steady-state growth. 
jumps at all points in time, and the conjectured answer would be wrong. The unique value of $\lambda$ that delivers this correspondence follows immediately as

$$
\lambda=\frac{1}{\Omega}=\frac{1}{\int_{0}^{\infty} e^{-[\rho v+\phi(v)]} d v} .
$$

To summarize, the solution for the household's consumption problem under log utility is that $c(t)$ be set as the fraction $\lambda$ of wealth at each date, where $\lambda$ is the constant shown in equation (13). The solution is time consistent because, if $c(t)$ is chosen in this manner at all future dates, then it will be optimal for consumption to be set this way at the current date. ${ }^{10}$

Inspection of equation (13) reveals that $\lambda=\rho$ in the standard Ramsey case in which $\phi(v)=0$ for all $v$. To assess the general implications of $\phi(v)$ for $\lambda$, it is convenient to rewrite equation (13) as

$$
\lambda=\frac{\boldsymbol{\int}_{0}^{\infty} e^{-[\rho v+\phi(v)]} \cdot\left[\rho+\phi^{\prime}(v)\right] d v}{\int_{0}^{\infty} e^{-[\rho v+\phi(v)]} d v} .
$$

Since the numerator of equation (14) equals unity, ${ }^{11}$ this result corresponds to equation (13).

The form of equation (14) is useful because it shows that $\lambda$ is a time-invariant weighted average of the instantaneous rates of time preference, $\rho+\phi^{\prime}(v)$. Since $\phi^{\prime}(v) \geq 0, \phi^{\prime \prime}(v) \leq 0$, and $\phi^{\prime}(v) \rightarrow 0$ as $v \rightarrow \infty$, it follows that

$$
\rho \leq \lambda \leq \rho+\phi^{\prime}(0) .
$$

10. This approach derives equation (13) as a Cournot-Nash equilibrium but does not show that the equilibrium is unique. Uniqueness is easy to demonstrate in the associated discrete-time model with a finite horizon, as considered by Laibson [1996]. In the final period, the household consumes all of its assets, and the unique solution for each earlier period can be found by working backwards sequentially from the end point. This result holds as long as $u(c)$ is concave, not just for log utility. The uniqueness result also holds if the length of a period approaches zero (to get continuous time) and if the length of the horizon becomes arbitrarily large. However, Laibson [1994] uses an explicitly game-theoretic approach to demonstrate the possibility of nonuniqueness of equilibrium in the infinite-horizon case. The existence of multiple equilibria depends on punishments that sanction past departures of consumption choices from designated values, and these kinds of equilibria unravel if the horizon is finite. My analysis of the infinite-horizon case does not consider these kinds of equilibria.

11. Use the change of variable $z=e^{-[p v+\phi(v)]}$. 
That is, $\lambda$ is intermediate between the long-run rate of time preference, $\rho$, and the short-run, instantaneous rate, $\rho+\phi^{\prime}(0)$.

The determination of the effective rate of time preference can be quantified by specifying the form of $\phi(v)$. Laibson [1997a] proposes a "quasi-hyperbola" in discrete time, whereby $\phi(v)=0$ in the current period and $e^{-\phi(v)}=\beta$ in each subsequent period, where $0<\beta \leq 1$. (Phelps and Pollak [1968] also use this form.) In this specification the discount factor between today and tomorrow includes the factor $\beta \leq 1$. This factor does not enter between any two adjacent future periods. Laibson argues that $\beta$ would be substantially less than one on an annual basis, perhaps between one-half and two-thirds.

This quasi-hyperbolic case can be applied to a continuoustime setting by specifying

$$
\phi(v)=0 \text { for } 0 \leq v \leq V, \quad e^{-\phi(v)}=\beta \text { for } v>V
$$

for some $V>0$, where $0<\beta \leq 1$. (In this specification, $\phi^{\prime}(v)$ is infinite at $v=V$ and equals zero otherwise.) Laibson's suggestion is that $V$ is small, so that the condition $\rho V \ll 1$ would hold.

Substitution from equation (16) into the formula for the integral in equation (12) leads to

$$
\Omega=(1 / \rho) \cdot\left[1-(1-\beta) \cdot e^{-\rho V}\right] .
$$

As $V$ approaches infinity, $\Omega$ goes to $1 / \rho$, which corresponds to the Ramsey case. The condition $\rho V \ll 1$ implies that the expression for $\Omega$ simplifies, as an approximation, to $\beta / \rho$, so that

$$
\lambda \approx \rho / \beta .
$$

If $\beta$ is between one-half and two-thirds, then $\lambda$ is between $1.5 \rho$ and $2 \rho$. Hence, if $\rho$ is 0.02 per year, then the heavy near-term discounting of future utility converts the Ramsey model into one in which the effective rate of time preference, $\lambda$, is $0.03-0.04$ per year.

The specification in equation (16) yields simple closed-form results, but the functional form implies an odd discrete jump in $e^{-\phi(v)}$ at the time $V$ in the future. More generally, the notion from the literature on short-term impatience is that $\rho+\phi^{\prime}(v)$ is high when $v$ is small and declines, say toward $\rho$, as $v$ becomes large. A simple functional form that captures this property in a smooth fashion is

$$
\phi^{\prime}(v)=b e^{-\gamma v}
$$


where $b=\phi^{\prime}(0) \geq 0$ and $\gamma>0$. The parameter $\gamma$ determines the constant rate at which $\phi^{\prime}(v)$ declines from $\phi^{\prime}(0)$ to zero.

Integration of the expression in equation (18), together with the boundary condition $\phi(0)=0$, leads to an expression for $\phi(v)::^{12}$

$$
\phi(v)=(b / \gamma) \cdot\left(1-e^{-\gamma v}\right) .
$$

this result can be substituted into the integral in equation (12) to get an expression for $\Omega$ :

$$
\Omega=e^{-(b / \gamma)} \cdot \int_{0}^{\infty} e^{\left[-\rho v+(b / \gamma) \cdot e^{-\gamma v}\right]} d v .
$$

The integral cannot be solved in closed form but can be evaluated numerically if values are specified for the parameters $\rho, b$, and $\gamma$.

To accord with Laibson's [1997a] observations, the parameter $b=\phi^{\prime}(0)$ must be around 0.50 per year, and the parameter $\gamma$ must be at least 0.50 per year, so that $\phi^{\prime}(v)$ gets close to zero a few years in the future. With $\rho=0.02, b=0.50$, and $\gamma=0.50, \Omega$ turns out to be 19.3 , so that $\lambda=1 / \Omega=0.052$. If $b=0.25$ and the other parameters are the same, then $\Omega=31.0$, and $\lambda=0.032$. Thus, the more appealing functional form in equation (19) has implications that are similar to those of equation (16).

The introduction of the $\phi(\cdot)$ term in the utility function of equation (2) and the consequent shift to a time-inconsistent setting amount, under log utility, to an increase in the rate of time preference above $\rho$. Since the effective rate of time preference, $\lambda$, is constant, the dynamics and steady state of the model take exactly the same form as in the Ramsey framework. The higher rate of time preference corresponds to a higher steady-state interest rate,

$$
r^{*}=\lambda \text {, }
$$

and, thereby, to a lower steady-state capital intensity, $k^{*}$, which is determined from the condition,

$$
f^{\prime}\left(k^{*}\right)=\lambda+\delta .
$$

Since the effective rate of time preference, $\lambda$, is constant, the model with log utility and no commitment is observationally equivalent to the conventional neoclassical growth model. That is, the equilibrium coincides with that in the standard model for a suitable choice of $\rho$. Since the parameter $\rho$ cannot be observed

12. The expression in equation (19) is similar to the "generalized hyperbola" proposed by Loewenstein and Prelec [1992, p. 580]. Their expression can be written as $\phi(v)=(b / \gamma) \cdot \log (1+\gamma v)$. 
directly, there is a problem in inferring from data whether the instantaneous rate of time preference includes the nonconstant term $\phi^{\prime}(v)$.

Some identification would be possible if different population groups-perhaps different countries-included time-varying timepreference rates to varying extents. More promising, however, is the idea that different societies have different technologies for committing their choices of future consumption. This element was missing in the model considered in this section-where no commitment ability existed-but is included in a later section.

\section{Population Growth and Technological Progress}

It is straightforward to incorporate population growth in the manner usually applied to the neoclassical growth model. If population (extended family size) grows at the constant rate $n$, then the utility function is assumed to be modified from equation (2) to

$$
U(\tau)=\int_{\tau}^{\infty} u[c(t)] \cdot e^{n t} \cdot e^{-[\rho \cdot(t-\tau)+\phi(t-\tau)]} d v .
$$

That is, the per capita flow of utils, $u[c(t)]$, is multiplied by the family size $e^{n t}$ (where the size at time 0 is normalized to unity). We assume, as is usual for the Ramsey model, that $\rho>n$, so that the net exponential term in equation (21) is guaranteed to be declining in $t$.

The solution under log utility is similar to that from before, except that the integral $\Omega$ is now defined by

$$
\Omega \equiv \int_{0}^{\infty} e^{-[(\rho-n) v+\phi(v)]} d v .
$$

The relation between the propensity to consume out of wealth, $\lambda$, and the modified $\Omega$ term is given by

$$
\lambda=n+(1 / \Omega),
$$

and the steady-state interest rate is again $r^{*}=\lambda$.

In the Ramsey case, where $\phi(v)=0$ for all $v, \Omega=1 /(\rho-n)$ (in equation (22)) and $\lambda=\rho$ (in equation (23)). For Laibson's quasi-hyperbolic preferences in equation (16), the result is

$$
\Omega \approx \frac{\beta}{\rho-n}, \quad \lambda \approx\left(\frac{\rho}{\beta}\right)-n \cdot \frac{1-\beta}{\beta} .
$$


If $0<\beta<1$, an increase in $n$ lowers $\lambda$ and, therefore, reduces the steady-state interest rate $r^{*}$.

It is also straightforward to introduce the conventional type of exogenous, labor-augmenting technological progress at the rate $x \geq 0$. The solution for $\lambda$ is still that shown in equations (22) and (23). However, since consumption per person grows in the steady state at the rate $x$, the condition for the steady-state interest rate under quasi-hyperbolic preferences is

$$
r^{*}=\lambda+x \approx(\rho / \beta)-n \cdot(1-\beta) / \beta+x .
$$

Hence, $r^{*}$ responds one-to-one to the rate of technological progress, $x$.

\section{The Role of Commitment}

With a constant rate of time preference, the results are the same whether the path of $c(t)$ is committed or not. With variable time-preference rates, the results depend on commitment technologies. Hence, the Laibson-style extension yields predictions about how commitment ability will affect the equilibrium. The extension also has significant welfare implications about the desirability of institutions and policies that facilitate commitments.

Laibson [1997a] discusses institutional mechanisms and markets that influence a household's ability to commit its consumption choices. Some of these mechanisms involve credit markets. For example, tax policies may subsidize saving through pensions and then impose penalties on early withdrawals. In private loan markets, difficulties in using future labor income as collateral work similarly to constrain spending. Public policies with respect to defaults affect the nature of this collateral. For example, lenient bankruptcy laws and the abolition of debtors' prisons reduce the value of future labor income as collateral and, thereby, provide some restraint on debt-financed consumer spending. From this perspective, improvements in lending technologies, such as the development of efficient mortgage markets for personal residences and an enhanced ability to borrow against human capital, reduce the extent of commitments. Usury laws and other legal constraints on credit markets may perhaps also be understood as crude public policies to inhibit excessive consumer spending through borrowing.

Laibson [1997a] stresses the negative effect on consumer 
commitment from improved liquidity. Recent changes that enhanced this liquidity include the increased availability of credit cards for purchases and borrowing and the expansion of ATM machines.

Some public policies outside of the financial markets can also be interpreted as commitment devices. This perspective may apply, for example, to legal prohibitions on gambling, drugs, and prostitution, which are forms of consumer expenditure that seem especially subject to short-run impatience.

Contracts can be used to constrain future spending, for example, by limitations on the breaking up of an estate by one's heirs. Changes in the enforceability of such contracts would influence commitment ability.

Durable goods that are hard to resell-such as houses and children-also provide some commitment on future consumption. However, durable purchases tend to set a floor on future consumption, and the household is usually more interested in setting a ceiling.

Some degree of commitment applies when consumption activities require advance planning, as in reservations for travel, theater, and restaurants. Improvements in transactions technologies, which reduce the required amount of advance planning, would have the side effect of lowering commitment ability.

Commitment can also be provided through personal discipline and self-control. The extent of this discipline may differ for cultural reasons across societies, but such differences are hard to quantify. Religious principles are dedicated, in part, toward curbing lavish expenditures and excessive debt. Thus, one potentially observable influence on commitment is religiosity, measured by church attendance or outlays on organized religion. Differences in affiliations may also matter because attitudes toward material pleasures differ across religions.

The effect of economic development on commitment ability is uncertain. On the one hand, improvements in financial markets and in the sophistication of contracts would allow people to make more binding commitments and, in that respect, expand their commitment capacity. On the other hand, the ability to commit would fall with enhanced liquidity of assets, expanded borrowing opportunities, and improvements in transactions technologies that reduce the required length of prior reservations. Other disturbances, such as wartime, may eliminate some prior commitments. 


\section{V.1. Full Commitment}

This subsection contrasts the no-commitment results with those under full commitment. In the latter regime, each household is assumed suddenly and unexpectedly to obtain the ability at date $\tau$ to commit choices of $c(t)$ for all future dates. Hence, the applicable first-order condition is given for $t>\tau$ from equation (5) by

$$
\left(\frac{1}{c}\right) \cdot\left(\frac{d c}{d t}\right)=r(t)-\rho-\phi^{\prime}(t-\tau) .
$$

The steady-state comparison between no and full commitment is straightforward. As already discussed, the no-commitment setting features a constant effective rate of time preference $\lambda \geq \rho$, as given in the setting with population growth by equations (22) and (23). In contrast, the full-commitment framework has an asymptotically constant rate of time preference of $\rho$. Therefore, the full commitment regime has lower $r^{*}$ and higher $k^{*}$ and $c^{*}$.

The short-run impact of introducing perpetual commitment is, however, opposite to the long-run effect. In the no-commitment situation the effective rate of time preference, $\lambda$, is an average of current and future instantaneous rates of time preference, as indicated in equation (14). ${ }^{13}$ With the introduction of commitment, the rate of time preference at date $\tau$ rises from $\lambda$ to $\rho+\phi^{\prime}(0)$. That is, the commitment ability initially makes households less patient. Then the effective rate of time preference declines steadily and asymptotically approaches $\rho \leq \lambda$.

The establishment of commitment motivates the household to substitute in favor of $c(\tau)$ in relation to $c(t)$ at all future dates. Under commitment, the time-preference factor that governs intertemporal substitution between $c(\tau)$ and $c(t)$ for $t>\tau$ is a weighted average of the instantaneous rates of time preference, $\rho+\phi^{\prime}(v)$, for $v$ running between 0 and $t-\tau$. This average approximates the no-commitment value, $\lambda$, for very large $t$. But, for small $t$, the weighted average relevant under commitment is a truncated version of $\lambda$. This truncation omits the most distantand, hence, smallest-instantaneous rates of time preference.

13. With population growth, the formula for $\lambda$ becomes

$$
\lambda-n=\frac{\boldsymbol{\int}_{0}^{\infty} e^{-[(\rho-n) v+\phi(v)]} \cdot\left[\rho-n+\phi^{\prime}(v)\right] d v}{\boldsymbol{\int}_{0}^{\infty} e^{-[(\rho-n) v+\phi(v)]} d v} .
$$


TABLE I

Full Commitment versus No Commitment

\begin{tabular}{lllllll}
\hline \hline & \multicolumn{1}{c}{$(1)$} & \multicolumn{1}{c}{$(2)$} & \multicolumn{1}{c}{$(3)$} & $(4)$ & \multicolumn{1}{c}{$(5)$} & $(6)$ \\
$\alpha$ & 0.75 & 0.75 & 0.75 & 0.33 & 0.33 & 0.33 \\
$b=\phi^{\prime}(0)$ & 0.50 & 0.20 & 0.10 & 0.50 & 0.20 & 0.10 \\
$\lambda$ & 0.0365 & 0.0248 & 0.0222 & 0.0365 & 0.0248 & 0.0222 \\
$\left(k_{\infty}^{*}-k_{0}^{*}\right) / k_{0}^{*}$ & 0.961 & 0.230 & 0.100 & 0.287 & 0.081 & 0.036 \\
$\left(c_{\infty}^{*}-c_{0}^{*}\right) / c_{0}^{*}$ & 0.265 & 0.061 & 0.026 & 0.021 & 0.005 & 0.002 \\
{$\left[c(0)_{\infty}-c(0)_{0}\right] / c(0)_{0}$} & 0.762 & 0.287 & 0.139 & 0.769 & 0.292 & 0.141 \\
$\begin{array}{c}\text { Compensating variation for com- } \\
\quad \text { mitment (as fraction of } k(0))\end{array}$ & 0.257 & 0.014 & 0.000 & 0.205 & 0.016 & 0.000 \\
\hline \hline
\end{tabular}

The subscript $\infty$ refers to perpetual commitment, and the subscript 0 to no commitment. All cases use the parameter values $\rho=0.02, \delta=0.05, n=0.01, x=0.02$, and $\gamma=0.50$. The starting capital stock $k(0)$ is set at 25 percent of the no-commitment steady-state value $k_{0}^{*}$.

Therefore, these truncated averages exceed $\lambda$. It follows that the introduction of full commitment at date $\tau$ raises $c(\tau) .{ }^{14}$ This rise in consumption is financed by forcing some future selves to save more than under no commitment. The ability to commit future saving is used enough so that $k(t)$ eventually rises above the no-commitment level. This higher capital stock applies in the long run despite the increase in $c(t)$ in the short run.

Table I contains quantitative results. Parameter values were specified, and numerical methods were used to work out the paths of $c(t), k(t), r(t)$, and other variables under no and full commitments. These simulations assume that all households have either no commitment ability or full commitment ability initiated at date zero. The results reflect a full general equilibrium, which allows for the determination of $r(t)$ and $w(t)$.

The table assumes in all cases the values $\rho$ (time-preference rate) $=0.02, \delta$ (depreciation rate) $=0.05, n$ (rate of population growth $)=0.01$, and $x$ (rate of technological progress $)=0.02$. The production function is assumed to be Cobb Douglas, $y=k^{\alpha}$, where $\alpha$ is taken to be either 0.75 (corresponding to a broad concept of

14. Since the introduction of commitment raises attained utility (as evaluated currently), there is also a positive income effect in the Hicksian sense. This effect reinforces the positive response of $c(\tau)$. Another way to assess the introduction of commitment is to note that the propensity to consume out of wealth-capital stock plus the present value of wages-is the same under no and full commitment (but only at the date $\tau$ at which commitment is introduced). Thus, the rise in $c(\tau)$ must correspond to a rise in the present value of wages. The problem with this perspective is that the rise in this present value, although correct, is not obvious. In the equilibrium, $w(t)$ falls, and $r(t)$ rises for some time after date $\tau$, and these patterns reverse only at some point in the future. 


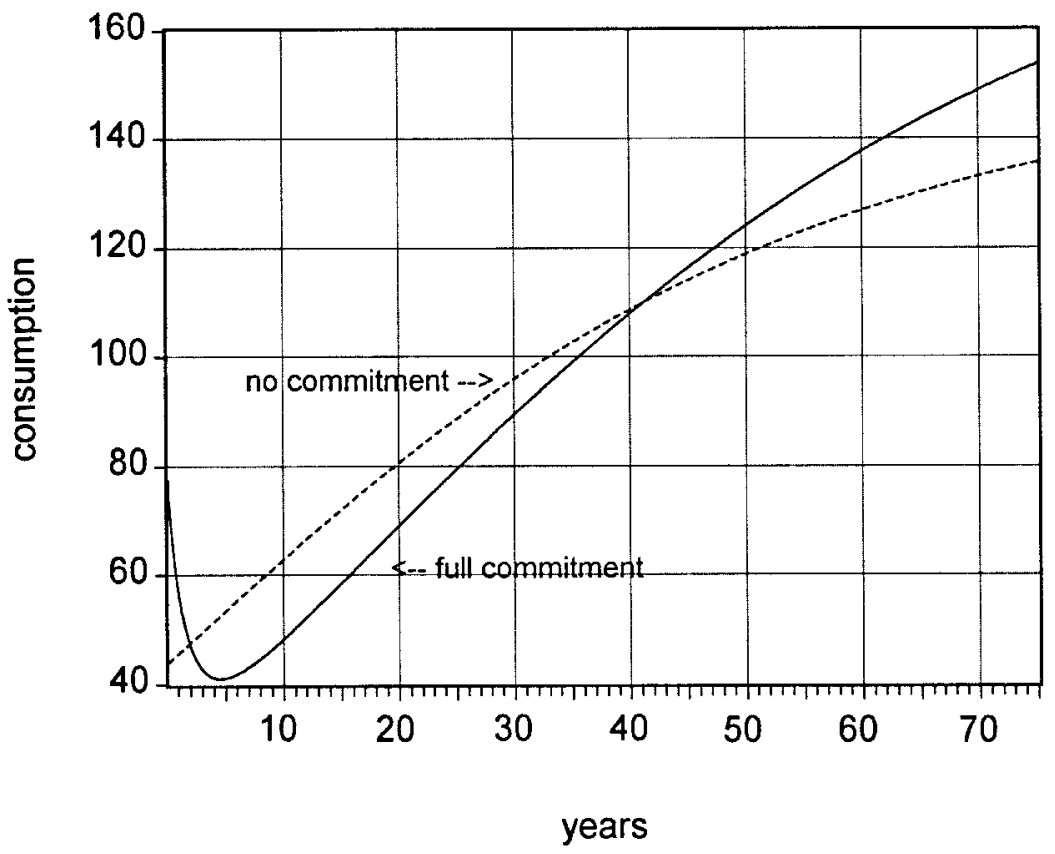

Figure Ia

Consumption-Full and No Commitment

capital) or 0.33 (corresponding to a conventional capital share). ${ }^{15}$ The $\phi$-function is given in equation (19), using the alternative values shown for $b=\phi^{\prime}(0)$. All of the simulations use the value $\gamma=$ 0.50 for the rate of decay of $\phi^{\prime}(t)$. The starting value $k(0)$ is assumed, in each case, to be 25 percent of the steady-state value, $k^{*}$, that applies under no commitment.

Consider the cases where $\alpha=0.75$. The value $b=\phi^{\prime}(0)=0.50$ implies a very high instantaneous rate of time preference in the short run. The effective rate of time preference with no commitment turns out to be $\lambda=0.0365$, and the steady-state values $k^{*}$ and $c^{*}$ under full commitment are, respectively, 96 percent and 26 percent above their no-commitment counterparts.

Figure I compares the paths for $c(t), s(t)$ (the gross saving rate), and $k(t)$ under no commitment with those under full commitment. In Figure Ia the establishment of perpetual commit-

15. With exogenous technological progress at the rate $x$, the values $y$ and $k$ represent quantities per unit of effective labor, $N(t) e^{x t}$, where $N(t)$ is population. 


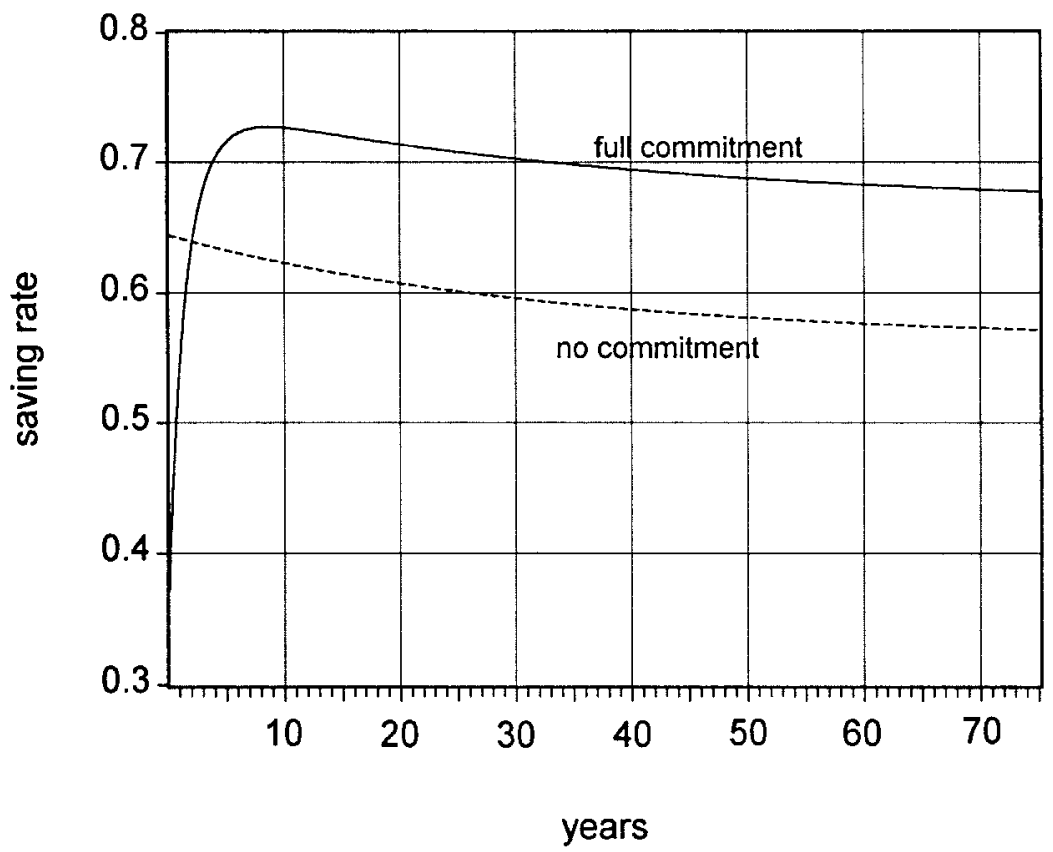

Figure Ib

Saving Rate-Full and No Commitment

ment at time zero raises $c(0) 76$ percent above the no-commitment value. The full-commitment $c(t)$ falls below the no-commitment level after 1.9 years, eventually grows faster than the nocommitment value, and permanently surpasses the no-commitment level after 41 years.

In Figure Ib the gross saving rate falls well below the no-commitment value in the short run but rises above this value after 2.1 years. This behavior dictates the path of the capital stock. Figure Ic shows that the full-commitment path of $k(t)$ falls for awhile below the no-commitment path but becomes permanently higher after 5.4 years.

These results generate empirically testable hypotheses that distinguish models with constant time-preference rates from those with variable rates. The testability relates to the presence or absence of institutional devices that allow households to commit future values of consumption. With constant time-preference rates, the existence of these devices does not matter. With 


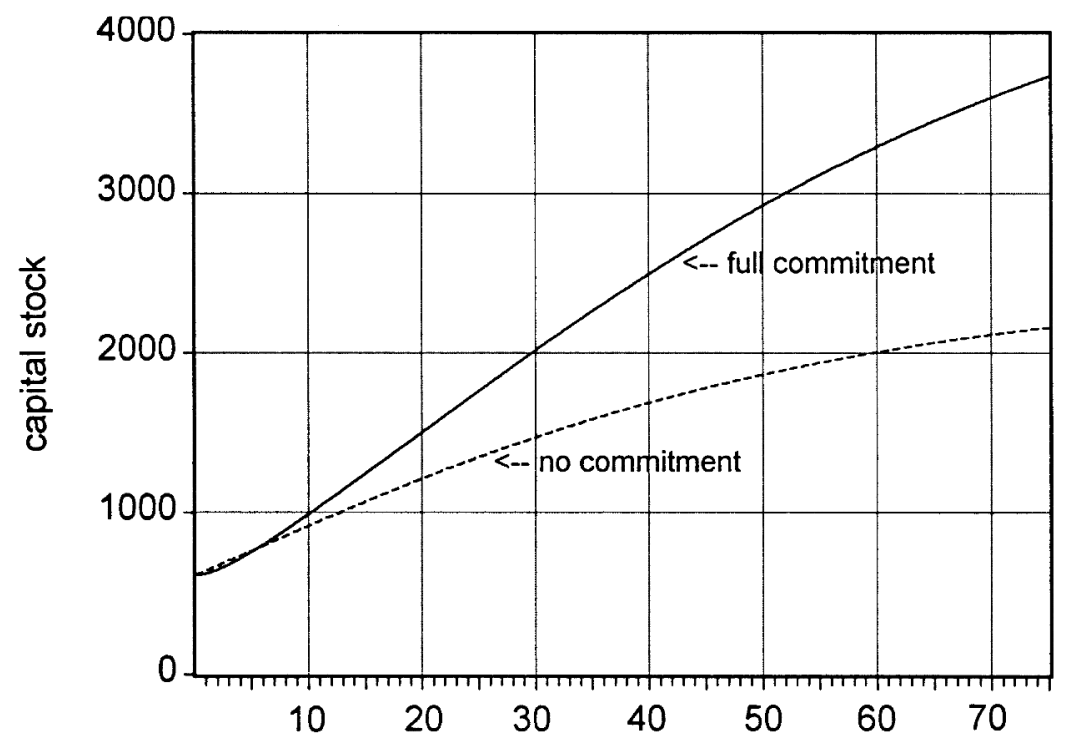

years

FIGURe Ic

Capital Stock-Full and No Commitment

Laibson-style preferences, the institutions can matter a great deal. In the long run the presence of commitment devices leads to more saving and capital accumulation. But more novel empirical implications relate to the dynamic effects from an institutional change. With Laibson-type preferences, the introduction of a commitment device first lowers saving and capital accumulation. These variables rise only after a significant delay.

The establishment of commitment at time zero raises attained utility, as evaluated from the perspective of households at that time (via equation (2)). One way to measure this improvement is by the compensating variation, which is the addition to the initial capital stock, $k(0)$, that no-commitment households would require to attain the same level of utility as fullcommitment households. For the case considered in column (1) of Table I, the compensating variation is 26 percent of the initial capital stock. ${ }^{16}$ Hence, with a very high instantaneous rate of time

16. The compensating variation is the amount of goods for which fullcommitment households would be just willing to relinquish their commitment 
preference in the short run $\left(b=\phi^{\prime}[0]=0.50\right)$, the value of commitment is substantial. ${ }^{17}$

Table I also contains results for less severe impatience in the short run. In column (2), where $b=\phi^{\prime}(0)=0.20$, full commitment raises $k^{*}$ and $c^{*}$ by only 23 percent and 6 percent, respectively, above the no-commitment values. The establishment of full commitment increases $c(0)$ by 29 percent, and the compensating variation for the commitment ability is only 1.4 percent of the initial capital stock.

In column (3), where $b=\phi^{\prime}(0)=0.10$, the responses are even smaller, and the compensating variation is essentially nil. Thus, if the decay rate for $\phi^{\prime}(t)$ is $\gamma=0.50$ per year, the value of commitment ability is significant only if the instantaneous rate of time preference is dramatically high in the short run. Even a value of $b$ as high as 0.20 per year generates a small value for commitment.

Columns (4)-(6) of Table I give the results for $\alpha=0.33$. The effects of commitment on $k^{*}$ and $c^{*}$ are much smaller than before: for example, in column (4) the increases are 29 percent and 2 percent, respectively, compared with 96 percent and 26 percent in column (1). The compensating variations for the commitment ability are lower in some cases-20 percent in column (4) versus 26 percent in column (1); but higher in others-1.6 percent in column (5) versus 1.4 percent in column (2).

\section{V.2. Partial Commitment}

The assumption here is that personal discipline or some institutional mechanism enables households to make imperfect commitments of future consumption. The specific modeling device

ability. A more interesting measure is the equivalent variation, the amount by which the initial capital stock of full-commitment households could be reduced to yield the same utility level achieved by no-commitment households. The equivalent variation measures the amount that no-commitment households would be willing to pay to establish perpetual commitment at time zero. Although the equivalent variation is more interesting, the compensating variation turns out to be much easier to calculate numerically. In practice, the two measures tell a similar story. For example, for the case considered in the first column of Table I, the equivalent variation is 21 percent of the initial capital stock, compared with 26 percent for the compensating variation.

17. Laibson [1997a, p. 467] computes compensating variations of comparable orders of magnitude for a case of partial commitment generated by the existence of illiquid assets. His analysis assumes that time is discrete with a period of one year and that the $\phi$-function takes the quasi-hyperbolic form of equation (16). 
is that households can select at date $\tau$ the consumption flows for a period of length $T \geq 0$, that is, over the closed interval $[\tau, \tau+T]$. However, these choices must respect any commitments that were made earlier.

Suppose, first, that the partial-commitment ability has been present at least since date $\tau-T$. In this case, a household at date $\tau$ will be selecting only $c(\tau+T)$. The choices of consumption in the half-open interval $[\tau, \tau+T$ ) will already have been made and committed beforehand. This problem is formally analogous to the one considered earlier, but new results emerge concerning the effect of the commitment interval, $T$.

The method for finding the optimal choice of $c(\tau)$ is analogous to that used before, when $T=0$, and only the results will be presented here. With log utility, the propensity to consume out of wealth is again constant, but the size of this propensity, denoted by $\lambda_{T}$, depends on $T$. The formula for $\lambda_{T}$ can be written as

$$
\lambda_{T}=e^{-[\rho T+\phi(T)] /} \Omega_{T},
$$

where the integral $\Omega_{T}$ is given by

$$
\Omega_{T} \equiv \int_{T}^{\infty} e^{-[\rho v+\phi(v)]} d v .
$$

For $T=0$ the results in equations (25) and (26) coincide with the one in equation (13), where recall that $\lambda_{0} \geq \rho$. It can be shown that $\lambda_{T}$ declines monotonically from $\lambda_{0}$ to $\rho$ as $T$ rises from zero to infinity. Thus, countries (or families) with better commitment technologies, as represented by higher values of $T$, have lower effective rates of time preference and, therefore, lower propensities to consume and higher propensities to save and accumulate capital.

Shifts in the ability to commit-modeled as changes in $T$-create transition intervals from one ongoing commitment situation to another. To illustrate the nature of these transitions, suppose that the ability to commit is initially nil $(T=0)$ and that people anticipate that this ability will always remain nil. Then the effective rate of time preference is the value $\lambda_{0}$, as derived before, which satisfies the inequality,

$$
\rho \leq \lambda_{0} \leq \rho+\phi^{\prime}(0) .
$$

Suppose that a commitment ability of length $T$ is introduced as a surprise at date $\tau$ and that everyone then believes that this regime will remain in place forever. At the outset the household 
can choose $c(t)$ over the interval $[\tau, \tau+T]$. In this context the usual first-order condition for consumption growth, as shown in equation (5), applies; that is,

$$
\left(\frac{1}{c}\right) \cdot\left(\frac{d c}{d t}\right)=r(t)-\rho-\phi^{\prime}(t-\tau)
$$

for $\tau<t<\tau+T$. This condition holds because, with the introduction of the commitment technology, the household can carry out the perturbations to the consumption path that underlie the condition.

At time $\tau$ the effective rate of time preference shifts discretely from $\lambda_{0}$ to the higher value $\rho+\phi^{\prime}(0)$. The effective rate of time preference then declines gradually to reach $\rho+\phi^{\prime}(T)$ at time $T$. At this point, the system returns to the case of ongoing commitment that has already been analyzed, and the effective rate of time preference shifts discretely downward to the value $\lambda_{T}$. The long-term effect is a lowering of the effective rate of time preference; that is, $\lambda_{T} \leq \lambda_{0}$.

The introduction of the commitment technology produces a discrete shift in the level of consumption. This effect has already been analyzed for the case $T=\infty$ in Figure Ia. Consumption increases mostly because of intertemporal substitution effects that favor current over future consumption.

The impact on current consumption is greater the larger the value of $T$. For example, Figure II shows the effects from the introduction of commitment level $T=1$ year at time zero. This case, treated in column (1) of Table II, assumes the parameter values $\alpha=0.75$ and $b=\phi^{\prime}(0)=0.50$. Consumption rises on impact by 25 percent of the no-commitment level, compared with 76 percent for $T=\infty$. In practice, a value $T=5$ is sufficient to get close to the $T=\infty$ results. That is, with a decay rate of $\gamma=0.50$ per year for $\phi^{\prime}(t)$, the ability to commit for five years is nearly equivalent to the ability to commit forever. For $T=5$ the rise in initial consumption is by 69 percent of the no-commitment value.

Figure II shows the full path of $c(t)$ for $T=1$. This partialcommitment path lies above the full-commitment value for $t$ between 2.9 and 52.1 years. In the long run the partialcommitment level of $c(t)$ is below the full-commitment value and above the no-commitment value.

The value of establishing partial commitment can be assessed in a manner analogous to that for full commitment. For the case displayed in Figure II (and in Table II, column (1)), the compensat- 


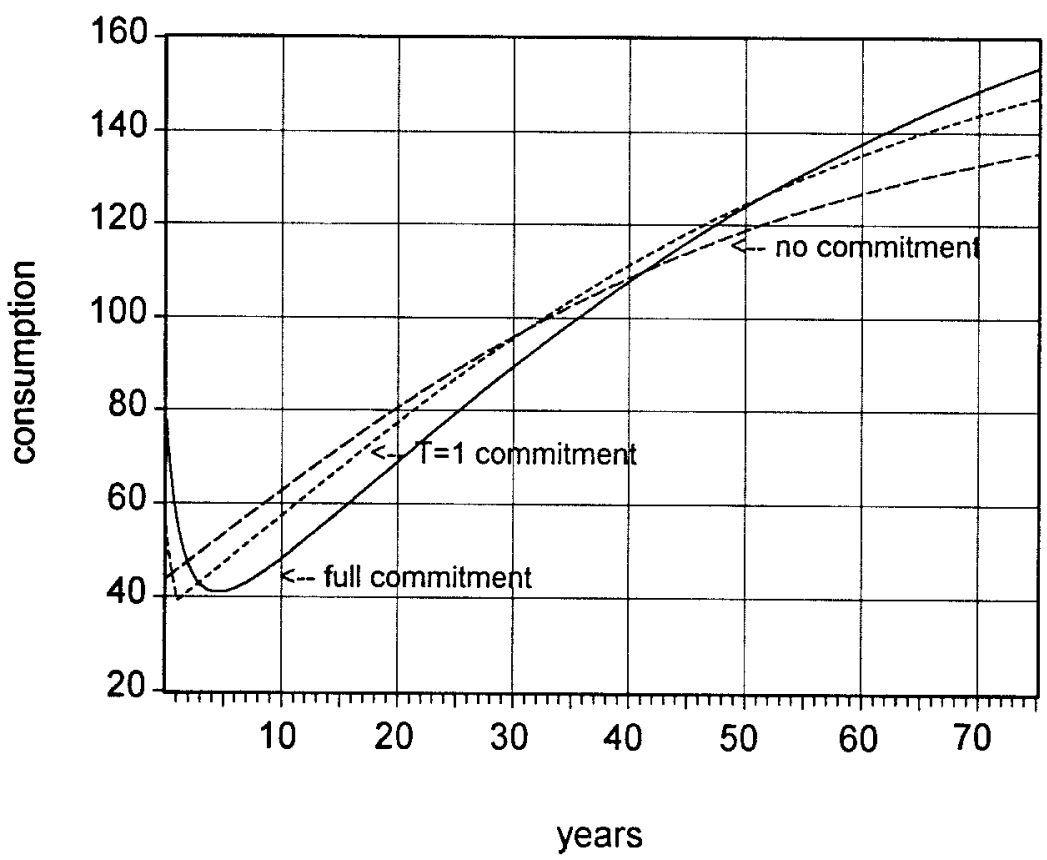

FIGURE II

Consumption-Full, Partial, and No Commitment

ing variation for $T=1$ commitment, compared with no commitment, is 19 percent of the initial capital stock. This value compares with 26 percent for full commitment. Hence, $T=1$ commitment goes a long way toward achieving the returns from perpetual commitment. Moreover, the results in column (1) of Table II show that $T=5$ commitment is worth virtually the same as perpetual commitment. ${ }^{18}$

Also as before, the value of commitment is much smaller if more moderate values are assumed for the instantaneous rate of time preference in the short run. With $b=0.20$ in column (2) of Table II, the compensating variation for $T=1$ is only 1.3 percent of the initial capital stock, compared with 1.4 percent for full or five-year commitment.

The results in this section show that commitment ability can

18. For $T=1$ the equivalent variation is 16 percent, compared with 19 percent for the compensating variation. For $T=5$ the equivalent variation is 21 percent, compared with 26 percent for the compensating variation. 
TABLE II

PaRtial Commitment

\begin{tabular}{llllllc}
\hline \hline & \multicolumn{1}{c}{$(1)$} & \multicolumn{1}{c}{$(2)$} & \multicolumn{1}{c}{$(3)$} & \multicolumn{1}{c}{$(4)$} & \multicolumn{1}{c}{$(5)$} & $(6)$ \\
$\alpha$ & 0.75 & 0.75 & 0.75 & 0.33 & 0.33 & 0.33 \\
$b=\phi^{\prime}(0)$ & 0.50 & 0.20 & 0.10 & 0.50 & 0.20 & 0.10 \\
$\lambda_{1}$ & 0.0281 & 0.0227 & 0.0213 & 0.0281 & 0.0227 & 0.0213 \\
$\left(k_{1}^{*}-k_{0}^{*}\right) / k_{0}^{*}$ & 0.390 & 0.094 & 0.040 & 0.138 & 0.034 & 0.015 \\
$\left(c_{1}^{*}-c_{0}^{*}\right) / c_{0}^{*}$ & 0.138 & 0.028 & 0.011 & 0.009 & 0.002 & 0.001 \\
{$\left[c(0)_{1}-c(0)_{0}\right] / c(0)_{0}$} & 0.250 & 0.109 & 0.055 & 0.347 & 0.136 & 0.067 \\
Compensating variation for partial & & & & & & \\
$\quad \begin{array}{l}\text { commitment } T=1 \text { (as fraction } \\
\quad \text { of } k[0])\end{array}$ & 0.187 & 0.013 & 0.000 & 0.126 & 0.010 & 0.000 \\
$\lambda_{5}$ & 0.0208 & 0.0203 & 0.0202 & 0.0208 & 0.0203 & 0.0202 \\
$\left(k_{5}^{*}-k_{0}^{*}\right) / k_{0}^{*}$ & 0.889 & 0.213 & 0.092 & 0.269 & 0.075 & 0.033 \\
$\left(c_{5}^{*}-c_{0}^{*}\right) / c_{0}^{*}$ & 0.253 & 0.057 & 0.024 & 0.020 & 0.005 & 0.002 \\
{$\left[c(0)_{5}-c(0)_{0}\right] / c(0)_{0}$} & 0.693 & 0.265 & 0.129 & 0.741 & 0.280 & 0.135 \\
Compensating variation for partial & & & & & & \\
$\quad$ commitment $T=5$ (as fraction & & & & & & \\
$\quad$ of $k(0))$ & 0.257 & 0.014 & 0.000 & 0.205 & 0.016 & 0.000 \\
\hline \hline
\end{tabular}

The subscripts 0,1 , and 5 refer to the commitment interval $T$. All cases use the parameter values $\rho=0.02$, $\delta=0.05, n=0.01, x=0.02$, and $\gamma=0.50$. The starting capital stock $k(0)$ is set at 25 percent of the no-commitment steady-state value $k_{0}^{*}$.

be viewed in a relative sense, represented by the parameter $T$, and does not have to be limited to the polar cases of no and full commitment. If we can observe institutional or cultural features that govern the extent of this ability, then the model generates empirically testable hypotheses. An expansion of commitment ability - a rise in $T$-raises the propensity to save in the long run. However, the short-run effects go in the opposite direction.

\section{Steady-State ANALYsis Under General Utility}

Suppose that households have isoelastic utility, as in equation (4):

$$
u(c)=\left(c^{1-\theta}-1\right) /(1-\theta),
$$

where $\theta>0$. Even with a constant rate of time preference, consumption is not a constant fraction of wealth unless $\theta=1$ (log utility). However, the first-order condition for consumption growth is well-known to take the simple form,

$$
\left(\frac{1}{c}\right) \cdot\left(\frac{d c}{d t}\right)=\left(\frac{1}{\theta}\right) \cdot[r(t)-\rho] .
$$


A reasonable conjecture is that the form of equation (27) would still hold when the household's objective includes a variable rate of time preference, as in equation (2). The presumptionbased on an extrapolation of the results under log utility-is that the constant $\rho$ would be replaced by some other constant that represented the effective rate of time preference. This conjecture is incorrect. The reason is that the effective rate of time preference at time $\tau$ involves an interaction of the path of the future values of $\phi^{\prime}(t-\tau)$ with future interest rates and, hence, is not constant when interest rates are changing (unless $\theta=1$ ). Some details of this interaction are worked out in the next section.

Although the transitional dynamics are complicated, it is straightforward to work out the characteristics of the steady state under a general concave utility function, $u(c)$. The key point is that, in a steady state, an increase in household assets would be used to raise consumption uniformly in future periods. This property makes it easy to compute propensities to consume for future periods with respect to current assets and, therefore, makes it easy to find the first-order optimization condition for current consumption. Only the results are presented here.

In the steady state (in the setting with zero population growth and technological progress), the interest rate is given by

$$
r^{*}=\frac{1}{\Omega}=\frac{1}{\int_{0}^{\infty} e^{-[\rho v+\phi(v)]} d v},
$$

where the integral $\Omega$ is given from equation (12) by

$$
\Omega=\int_{0}^{\infty} e^{-[p v+\phi(v)]} d v .
$$

Thus, the steady-state interest rate under a general concave utility function coincides with that for log utility, as shown in equation (13).

VII. TRansitional Behavior Under Isoelastic Utility

Assume again isoelastic utility:

$$
u(c)=\left(c^{1-\theta}-1\right) /(1-\theta),
$$

where $\theta>0$. Overall utility can, as before, be broken up into a part 
from $\tau$ to $\tau+\varepsilon$ and another part from $\tau+\varepsilon$ to infinity:

$$
U(\tau) \approx \varepsilon \cdot \frac{\left[c(\tau)^{1-\theta}-1\right]}{(1-\theta)}+\int_{\tau+\varepsilon}^{\infty} \frac{\left[c\left(t^{1-\theta}\right)-1\right]}{(1-\theta)} \cdot e^{-[(\rho \cdot(t-\tau)+\phi(t-\tau)]} d t .
$$

The effect of $c(\tau)$ on the assets, $k(\tau+\varepsilon)$, available at date $\tau+\varepsilon$ is still given from equation (9) by

$$
d[k(\tau+\varepsilon)] / d[c(\tau)] \approx-\varepsilon .
$$

The difficult part of the problem is the assessment of the response of $c(t)$ to $k(\tau+\varepsilon)$ for $t \geq \tau+\varepsilon$. This response can be measured by modifying the form of the conjectured solution for $c(t)$ from that in equation (10) to

$$
\left(\frac{1}{c}\right) \cdot\left(\frac{d c}{d t}\right)=\left(\frac{1}{\theta}\right) \cdot[r(t)-\lambda(t)]
$$

for $t \geq \tau+\varepsilon$. This specification allows $\lambda(t)$ to vary over time and, therefore, is restrictive only in that $\lambda(t)$ does not depend on the level of initial assets. This conjecture implies that the choices of consumption at date $\tau$ and, hence, of assets at date $\tau+\varepsilon$ affect the level but not the shape of the path of future consumption.

The analysis proceeds as under log utility, but with more algebra, to derive the first-order condition for the choice of $c(\tau)$, given that the behavior of future consumption accords with equation (30). Define time averages of $r(t)$ and $\lambda(t)$ by

$$
R(t, \tau) \equiv \frac{1}{t-\tau} \cdot \int_{\tau}^{t} r(v) d v, \quad \Lambda(t, \tau) \equiv \frac{1}{t-\tau} \cdot \int_{\tau}^{t} \lambda(v) d v .
$$

The optimization condition leads eventually (as $\varepsilon$ approaches zero) to

$$
\begin{aligned}
& \int_{\tau}^{\infty} e^{[(1-\theta) / \theta] \cdot R(t, \tau) \cdot(t-\tau)-(1 / \theta) \cdot \Lambda(t, \tau) \cdot(t-\tau)\}} \\
& \cdot\left\{e^{[\Lambda(t, \tau)-\rho] \cdot(t-\tau)} \cdot e^{-\phi \cdot(t-\tau)}-1\right\} d t=0 .
\end{aligned}
$$

This condition implies that $\Lambda(t, \tau)=\lambda(t)=\rho$ (the standard Ramsey result) if $\phi(t-\tau)=0$ for all $t \geq \tau$. Equation (31) reduces to equation (13) if $\theta=1$.

If we differentiate the left-hand side of equation (31) with respect to $\tau$ and set the result to zero, then we get, after 
simplifying,

$$
\lambda(\tau)=\frac{\int_{\tau}^{\infty} \omega(t, \tau) \cdot\left[\rho+\phi^{\prime}(t-\tau)\right] d t}{\int_{\tau}^{\infty} \omega(t, \tau) d t},
$$

where $\omega(t, \tau)$ is a weighting factor, given by

$$
\omega(t, \tau)=e^{[(1-\theta) / \theta] \cdot[R(t, \tau)-\Lambda(t, \tau)] \cdot(t-\tau)} \cdot e^{-[\rho \cdot(t-\tau)+\phi(t-\tau)]} .
$$

Hence, $\lambda(\tau)$ is a weighted average of future instantaneous rates of time preference (as viewed from time $\tau$ ), $\rho+\phi^{\prime}(t-\tau)$. Equation (14) is the special case of equations (32) and (33) for $\theta=1$.

The weight $\omega(t, \tau)$ reflects the sensitivity of $c(t)$ to $k(\tau+\varepsilon)$, expressed as a present value at date $\tau$. Equation (30) implies that $c(t)$ equals $c(\tau+\varepsilon)$ multiplied by the factor $e^{(1 / \theta) \cdot[R(t, \tau)-\Lambda(t, \tau)](t-\tau)}$. The present value factor is $e^{-R(t, \tau) \cdot(t-\tau)}$. The net effect from interest rates on $\omega(t, \tau)$ therefore appears as $e^{[(1-\theta) / \theta] \cdot R(t, \tau) \cdot(t-\tau)}$. This term is time varying unless $\theta=1$ or interest rates are constant.

If $\theta>1$-signifying that households are not very willing to substitute consumption intertemporally - then $\omega(t, \tau)$ declines with $R(t, \tau)$. If the economy begins with a capital intensity below its steady-state value, then $r(\tau)$ tends to start high and then fall toward its steady-state value. The weights $\omega(t, \tau)$ are then particularly low for dates $t$ far in the future. Since these dates are also the ones with relatively low values of $\rho+\phi^{\prime}(t-\tau), \lambda(\tau)$ is high initially. However, as $r(\tau)$ falls, the weights $\omega(t, \tau)$ become more even, and $\lambda(\tau)$ declines. This descending path of $\lambda(\tau)$ means that households effectively become more patient over time.

The effects are, however, reversed if $\theta<1$, a situation in which households are very willing to substitute consumption intertemporally. In this case, the net effects from interest rates are opposite to those just discussed.

The precise dynamics are difficult to work out because equations (32) and (33) express $\lambda(\tau)$ as a function of integrals of future values of $\lambda(t)$. It would probably be feasible to use numerical methods to simulate the economy's transition path.

\section{CONCLUDING OBSERVATIONS}

The allowance for variable rates of time preference leaves intact the basic properties of the neoclassical growth model. Consumption depends on an effective rate of time preference, which is a weighted 
average of future instantaneous rates. Under log utility the weights are constant, and the effective rate of time preference is constant. Therefore, in this case, the extended model is observationally equivalent to the standard one. Under more general specifications of utility, there are new results that involve the dynamic interplay between interest rates and effective rates of time preference.

Despite this correspondence in form, a variable rate of time preference can have quantitatively important implications for saving and growth-these effects are analogous to those generated in the standard model from differences in the rate of time preference. There are also potentially important welfare implications, because the outcomes in a no-commitment equilibrium can differ greatly from those that would arise if households were able fully to commit their future choices of consumption. However, simulations indicate that these effects are substantial only if short-term rates of time preference are very high-something like 0.50 per year. If these rates are below 0.20 per year and other parameters take on reasonable values, then the ability to commit is not worth a great deal.

From a positive standpoint, the most important macroeconomic predictions involve the relation between commitment technologies and the propensity to save. Economies that feature a greater capacity to commit future consumption have lower effective rates of time preference in the long run and, thereby, exhibit higher steady-state levels of saving and capital accumulation. However, the short-run effects from the introduction of commitment devices go in the opposite direction: saving and capital accumulation tend to decline for awhile if the commitment technology improves.

The key to the empirical analysis involves the isolation of institutional and other features that govern the degree of commitment. This commitment capacity involves partly the state of financial markets and the legal and tax systems. However, some changes that typically accompany economic development-such as a greater capacity to write enforceable contracts-would enhance the ability to commit, whereas others-such as increased liquidity of financial assets and improved functioning of credit markets-would go the other way. Additional explanatory power may come from cultural factors, such as religiosity, that influence the extent of personal self-control. 


\section{REFERENCES}

Ainslie, George W., Picoeconomics (Cambridge, Cambridge University Press, 1992).

Akerlof, George A., "Procrastination and Obedience," American Economic Review, Papers and Proceedings, LXXXI (May 1991), 1-19.

Barro, Robert J., and Xavier Sala-i-Martin, Economic Growth (New York: McGraw Hill, 1995).

Becker, Gary S., and Robert J. Barro, "A Reformulation of the Economic Theory of Fertility," Quarterly Journal of Economics, CIII (February 1988), 1-25.

Cass, David, "Optimum Growth in an Aggregative Model of Capital Accumulation," Review of Economic Studies, XXXII (July 1965), 233-240.

Epstein, Larry G., and J. Allan Hynes, "The Rate of Time Preference and Dynamic Economic Analysis," Journal of Political Economy, XCI (August 1983), 611635.

Fishburn, Peter C., and Ariel Rubinstein, "Time Preference," International Economic Review, XXIII (October 1982), 677-693.

Fisher, Irving, The Theory of Interest (New York: Macmillan, 1930).

Goldman, Steven M., "Consistent Plans," Review of Economic Studies, XXXXVII (April 1980), 533-537.

Koopmans, Tjalling C., "Stationary Ordinal Utility and Impatience," Econometrica, XXVIII (April 1960), 287-309.

—, "On the Concept of Optimal Economic Growth," in The Econometric Approach to Development Planning (Amsterdam, North-Holland, 1965).

Laibson, David, "Self-Control and Saving," unpublished, Harvard University, May 1994.

"Hyperbolic Discount Functions, Undersaving, and Savings Policy," NBER Working Paper No. 5635, June 1996.

, "Golden Eggs and Hyperbolic Discounting," Quarterly Journal of Economics, CXII (May 1997a), 443-477.

"Hyperbolic Discount Functions and Time Preference Heterogeneity," unpublished, Harvard University, March 1997b.

Loewenstein, George, and Drazen Prelec, "Anomalies in Intertemporal Choice: Evidence and an Interpretation," Quarterly Journal of Economics, CVII (May 1992), 573-598.

Phelps, Edmund S., and Robert A. Pollak, "On Second-Best National Saving and Game-Equilibrium Growth," Review of Economic Studies, XXXV (April 1968), 185-199.

Pollak, Robert A., "Consistent Planning," Review of Economic Studies, XXXV (April 1968), 201-208.

Ramsey, Frank, "A Mathematical Theory of Saving," Economic Journal, XXXVIII (December 1928), 543-559.

Strotz, Robert H., "Myopia and Inconsistency in Dynamic Utility Maximization," Review of Economic Studies, XXIII (1956), 165-180.

Thaler, Richard, "Some Empirical Evidence on Dynamic Inconsistency," Economics Letters, VIII (1981), 201-207. 